\title{
NY-ESO-I Protein Vaccine Combining Alum, CpG ODN, and HH2 Complex Adjuvant Induces Protective and Therapeutic Anti-Tumor Responses in Murine Multiple Myeloma
}

This article was published in the following Dove Press journal: OncoTargets and Therapy

\author{
Hao Wang' \\ Wei Huang ${ }^{2}$ \\ Hua Gao ${ }^{3}$ \\ Ting Ting Liu ${ }^{4,5}$ \\ 'Department of Surgery, The First \\ Affiliated Hospital of Chengdu Medical \\ College, Chengdu Medical College, \\ Chengdu, Sichuan, People's Republic of \\ China; ${ }^{2}$ Department of Preventive \\ Medicine, West China School of Public \\ Health, Sichuan University, Chengdu, \\ Sichuan, People's Republic of China; \\ ${ }^{3}$ Department of Hematology, The Third \\ People's Hospital of Chengdu, Chengdu, \\ Sichuan 61004I, People's Republic of \\ China; ${ }^{4}$ Department of Hematology, \\ Renji Hospital, School of Medicine, \\ Shanghai Jiaotong University, Shanghai \\ 200127, People's Republic of China; \\ ${ }^{5}$ Department of Hematology, West China \\ Medical Center, Sichuan University, \\ Chengdu, Sichuan 6I004I, People's \\ Republic of China
}

Background: NY-ESO-1 is an ideal target for multiple myeloma immunotherapy. Alum, CpG ODN and HH2 complex is a safe and effective adjuvant for cancer vaccine.

Methods: We constructed NY-ESO-1 protein vaccine combined with alum, CpG ODN, and $\mathrm{HH} 2$ complex adjuvant to immunize the BALB/c mice inoculated with NS-1 murine multiple myeloma cells. Then, we determined the immunogenicity and anti-tumor effects in prophylactic and therapeutic models by analyzing the NY-ESO-1 antibody titer, evaluating IL4/INF$\gamma$ expression, and assessing cytotoxic $\mathrm{T}$ lymphocytes activities. The side-effects of vaccines were also evaluated.

Results: The group of NY-ESO-1 protein vaccine combining alum, $\mathrm{CpG}$ ODN, and $\mathrm{HH} 2$ complex adjuvant is more capable of stimulating both humoral and cellular tumor-specific immune responses to prolong the survival of the mice and inhibit tumor growth in prophylactic and therapeutic immunotherapy. The marked side-effects were not detected in immunized mice.

Discussion: The results suggest that alum, $\mathrm{CpG}$ ODN, and $\mathrm{HH} 2$ complex as a novel immune adjuvant combined cancer vaccine could improve the immunity efficiency in a murine multiple myeloma model.

Keywords: vaccine, alum, $\mathrm{CpG} \mathrm{ODN}, \mathrm{HH} 2$, complex adjuvant, immunity, multiple myeloma

\section{Introduction}

Multiple myeloma (MM) arises from plasma cell malignancy and ranks as a second prevalent hematopoietic malignancy. ${ }^{1,2}$ So far, it's still an incurable disease, although the overall outcomes are constantly improving from high-dose chemotherapy, autologous stem-cell transplantation, and the introduction of novel agents (thalidomide, lenalidomide, bortezomib). ${ }^{2,5}$ The important cause of treatment failure is that the patients have poor tolerability to further intervention and myeloma cells have acquired resistance to previous therapy and very likely are cross-resistant to similar therapy. ${ }^{8,10}$ Therefore, it is urgent that some novel therapeutic intervention should be developed to eliminate myeloma cells that have become multi-drug resistant. Anti-tumor vaccine is regarded as an effective and reliable anti-tumor immune therapeutic measure because it is well-tolerated, non-toxic, and unlikely cross-resistant with current drugs, and thus might be a powerful intervention to
Correspondence: Ting Ting Liu Department of Hematology, Renj Hospital, School of Medicine, Shanghai Jiaotong University, 160 Pujian Road, Shanghai 200I27, People's Republic of China

Tel +86-|3882203| 24

Email liutingting_0I3I@sina.com 
maintain a long-lasting control of minimal residual disease (MRD) or to even eradicate disseminated tumor cells. ${ }^{2,3,5,7}$

It is critical to identify tumor specific antigens (TSAs) in the preparation of tumor vaccines. NY-ESO-1 is a member of the cancer/testis antigen (CTA) family expressed in diverse types of tumors including multiple myeloma, but not in normal adult tissues except for germ cells in testes and ovaries. ${ }^{11,15}$ Among all CTAs found so far, NY-ESO-1 is the most immunogenic ${ }^{14,16,19}$ and produces clinical responses in chemo-refractory cancers. The fairly high immunogenicity means NY-ESO-1 positive patients can produce humoral and cellular immune responses against NY-ESO-1. In addition, NY-ESO-1 protein vaccine combined with adjuvant can modulate the Th1/Th2 balance and increase the magnitude and durability of adaptive immunity. Therefore, NY-ESO-1 is an ideal target for MM immunotherapy.

The immune adjuvant is an effective way to enhance immunogenicity of cancer vaccine. Alum is the most common adjuvant, leading to antigen retention and prolonging antigen exposure. ${ }^{20,21}$ Alum can strengthen the antibody-mediated immune response with a Th2 bias, ${ }^{22,23}$ but is poor in $\mathrm{CD} 8^{+} \mathrm{T}$ cells generation. ${ }^{24} \mathrm{CpG}$ oligodeoxynucleotide (CPG ODN) is a specific nucleotide sequence with non-methylated cytosine guanine as the primitive unit. It activates a variety of immune cells including $\mathrm{B}$ cells, $\mathrm{T}$ cells, NK cells, macrophages, and dendritic cells by binding to TLR9 and it can increase Th1-type immune response and make up the lack of alum, considered as a type of promising adjuvant. ${ }^{25,27}$ VQLRIRVAVIRA-NH2 (HH2) is one type of innate defense regulator peptide (IDR) ${ }^{28}$ which can recruit DC, neutrophils, and macrophages indirectly or directly and stimulate production of cytokines (monocyte chemoattractant protein-1, MCP-1) and cytokines (IL-8, IFN- $\gamma$ ). ${ }^{29,30,32}$ A previous study indicated that alum-CpG ODN-HH2 complex was an effective and novel immune adjuvant. ${ }^{31}$

In our current study, we develop a complex vaccine based on NY-ESO-1-alum-CpG ODN-HH2 (named $\mathrm{NACH}$ ) and examine its anti-tumor effects and immunogenicity on prophylactic and therapeutic models of murine multiple myeloma. We found that alum, CpG ODN, and $\mathrm{HH} 2$ complex as novel immune adjuvant combined cancer vaccine could improved the immunity efficient in murine multiple myeloma model. Compared with others vaccine groups, the results showed that $\mathrm{NACH}$ vaccine could markedly improve the immunity efficient and anti-tumor activity in murine multiple myeloma models through inducing humoral and cellular tumor-specific immune responses. The results may provide an effective immunotherapy strategy for multiple myeloma.

\section{Materials and Methods Mice}

Female BALB/c mice (6-8 weeks old) were purchased from WeiTongLiHua Biological Technology Company (Beijing, China) and maintained at the Animal Center of State Key Laboratory of Biotherapy of Sichuan University. Our operation procedure obeys the protocols approved by the Ethics Review Committee for Animal Experimentation of Sichuan University. The approval number for the mouse model experiments is KS2020410.

\section{Cell Lines}

NS-1 murine multiple myeloma cells were provided by American Type Culture Collection (ATCC). Then they were transfected with the constructed plasmid pcDNA3.1/ NY-ESO-1 using Lipofec tamine 2000 (Invitrogen, Carlsbad, CA), fostered in 1640 medium with $10 \% \mathrm{FBS}$, and incubated in a 37 incubator with a humid $5 \% \mathrm{CO}_{2}$ gas environment. The NS-1 murine multiple myeloma cells can stably express NY-ESO-1 (NY-ESO- ${ }^{+}$NS-1), which was verified by Western blotting.

\section{NY-ESO-I Protein and Vaccine}

The production and purification for NY-ESO-1 protein were presented by the following steps. First, we assembled a bacterial expression plasmid PET32a (Invitrogen) and NY-ESO-1 (Invitrogen). Then we injected PET32a-NYESO-1 into the E. coli strain BL21 (DE3)-bearing to make it recombine, and induced it with IPTG for protein production. Finally we lysed the E. coli using a highpressure homogenizer (APV 2000, Lubeck, Germany) to attain crude NY-ESO-1 protein (Trx-NY-ESO-1). Here are four steps for the purification of NY-ESO-1 protein, which included Ni-chelating Sepharose affinity chromatography (GE Healthcare, Piscataway, NJ), excision of the Trx-His6 -tag, removal of the Trx-His6-tag with second Ni-chelating Sepharose affinity chromatography, and Q-ion-exchange chromatography (GE Healthcare, Piscataway, NJ). The endotoxin level was below standard level.

The preparation of combine vaccine needs the following steps. In short, $20 \mu \mathrm{g} \mathrm{CpG} \mathrm{ODN} \mathrm{was} \mathrm{mixed} \mathrm{with} 40 \mu \mathrm{g}$ $\mathrm{HH} 2$, and then combined with $125 \mu \mathrm{g}$ alum, next mixed with $5 \mu \mathrm{g}$ recombinant NY-ESO-1 protein in PBS with 
a total volume of $100 \mu \mathrm{L}$. The endotoxin levels were approximately $0.02 \mathrm{EU} / \mu \mathrm{g}$.

\section{Western Blot Analysis}

The final purified NY-ESO-1 proteins were detected by Western blot (WB) analysis. Briefly, the proteins were exposed to sodium dodecyl sulfate-polyacrylamide gel electrophoresis analysis. After proteins were transferred to nitrocellulose membrane and subsequent blocking, the membranes were immunoblotted with rabbit anti-mice NY-ESO-1 antibody and visualized with horseradish peroxidase (HRP)-conjugated goat anti-rabbit IgG antibody (Santa Cruz Biotechnology, Santa Cruz, CA, USA), followed by enhanced chemiluminescence (Pierce Biotechnology, Rockford, IL, USA) and auto-radiography.

\section{Mouse Models}

In the prophylactic model, female BALB/c mice $(n=10)$ were subcutaneously (s.c.) vaccinated with NY-ESO-1, NYalum-CpG-HH2 on days 0,14 , and 21 , respectively. The mice treated with NS were used as controls. Two weeks after the last immunization, NY-ESO- $1^{+} \mathrm{NS}-1\left(2 \times 10^{5}\right)$ cells were implanted on the back of mice and tumor volume was measured every 3 days from tumor onset. We adopt the following formula to calculate tumor volume: $0.5 \times$ length $(\mathrm{mm}) \times$ width $\left(\mathrm{mm}^{2}\right)$. At the same time, the murine survival was monitored and plotted as Kaplan Meier curves.

In the therapeutic model, mice were injected with NYESO- $1^{+}$NS- $1\left(2 \times 10^{5}\right)$ cells on day 0 as described above. Once the tumor became palpable $\left(>50 \mathrm{~mm}^{3}\right)$, these mice were randomly divided into three groups on average, which correspond to three immunization methods respectively as mentioned above. Then mice were immunized three times (on days 7, 14, and 28). The tumor volume and body weight were measured, and the murine survival was monitored and plotted as Kaplan-Meier curves.

\section{Analysis of NY-ESO-I Antibody Titers}

One week after the last immunization, we collected serum and detected NY-ESO-1 antibody titers by ELISA according to the manufacturer's instructions (FangChen Corporation, Beijing, China). Briefly, 3-fold diluted serum was analyzed on plates (Nunclon, Roskilde, Denmark) coated with NY-ESO-1 protein $(0.1 \mu \mathrm{g} /$ well $)$ overnight at $4^{\circ} \mathrm{C}$ followed by blocking with $5 \%$ non-fat dry milk and $0.05 \%$ Tween-20 in PBS. Then we probed NY-ESO-1 specific antibodies with goat antimouse IgG conjugated with horseradish peroxidase
(1:3000; ZSGB-BIO, Beijing, China). Finally, the absorbance of each well at A450 was read on an microplate reader (iMark, Bio-Rad, American).

\section{CTL Activities}

CTL assay was assessed using the standards lactate dehydrogenase (LDH) method according to the protocol (GMS 10073 V. A, GENMED, American). Murine splenocytes 1 week after the third immunization were isolated as effector cells, and the NS-1 mouse myeloma cells were regarded as target cells. NS-1 1 target cells were plated at $1 \times 104$ cells/ well on 96-well U- bottomed plates, and the splenocytes (effectors) were added to a final volume of $100 \mu \mathrm{L}$ in ratios of $1: 10,1: 20$, and 1:40 (target to effector). The plates were then incubated for 4 hours in a humidified $5 \%$ $\mathrm{CO}_{2}$ chamber at $37^{\circ} \mathrm{C}$ and centrifuged at $250 \mathrm{~g}$ for 10 minutes. Half of the volume $(50 \mu \mathrm{L})$ was moved from all wells to fresh 96-well at-bottom plates, and an equal volume of reconstituted substrate mix was added per well.

The plates were then incubated in the dark at room temperature for 30 minutes, then the $50 \mu \mathrm{L}$ stop solution added, and finally the absorbance was measured at 490 $\mathrm{nm}$. The cell death percentages at each effector-to-target cell ratio were calculated using the following formula: (A490nm (experimental)-A490nm (effector spontaneous)A490nm (target spontaneous)/(A490nm (target maximum)-A490nm (effector spontaneous)-A490nm (target spontaneous) $\times 100$.

\section{ELISA}

One week after the immunization, the immunized mice were sacrificed and the splenocytes were collected, and then ELISA assays were carried out using the mouse IFN$\gamma /$ IL-4 ELISA kit (Dakewe Biotech Company, Shenzhen, China) according to the manufacturer's instructions. Briefly, $5 \times 10^{5}$ splenocytes were isolated and then stimulated with NS-1 cell lysis buffer including NY-ESO-1 protein in $100 \mu \mathrm{L} /$ well RPMI 1640 supplemented with $10 \% \mathrm{FBS}$ at $37^{\circ} \mathrm{C}$ for 48 hours. The supernatants were collected and serial diluted standards and samples were seeded into 96-well plates. After adding reagents, washing, chromogenic development, and quenching were as described in the manual instruction of test kit. The absorbance value at $450 \mathrm{~nm}$ was measured for each well. A linear regression was performed based on the standards for further deducing the concentration of test samples. 


\section{Monitoring the Side Effects of Vaccines}

After the animals were sacrificed, the heart, liver, spleen, lung, and kidney of mice were gathered, embedded, and slided for histological examinations. By the way, the body weight, appetite, hair, and activity of mice were also measured during the whole immune procedure.

\section{Statistical Analysis}

All data was analyzed by GraphPad Prism6 statistical software (GraphPad Software, USA). We used one-way analysis of variance (ANOVA) followed by Tukey's multiple-range testing to compare difference of multiple groups. All values were depicted as the means \pm SD. $P<0.05$ was considered statistically significant.

\section{Results}

\section{Identification of Vaccines and Western Blot Assay}

After transfection of E. Coli Strain BL21 (DE3)-bearing and induction with IPTG, the protein expressed by PET32A-NY-ESO-1 was purified. Western blot assay revealed the purified NY-ESO-1 protein as a $18 \mathrm{KD}$ protein band, which could react with rabbit anti-mice NYESO-1 antibody (Figure 1).

\section{NACH Vaccine Inhibits Tumor Growth and Prolongs Survival Time of Mice in a Murine Multiple Myeloma Model}

In the prophylactic model (Figure 2A and B), the inoculated NS-1 mouse multiple myeloma grew rapidly in the control group, with a mean tumor volume of approximately $983 \pm 101.16 \mathrm{~mm}^{3}$ on day 17 . However, the mean tumor volume was $402.9 \pm 41.1 \mathrm{~mm}^{3}$ in the

\section{NY-ESO-1}

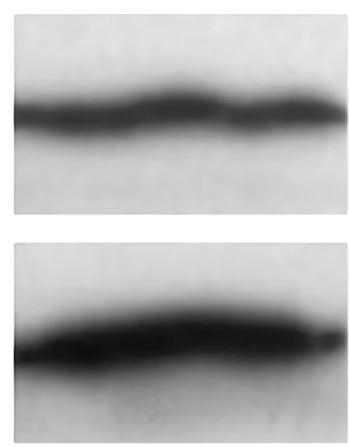

$18 \mathrm{KD}$

$\beta$-actin

Figure I Identification of NY-ESO-I protein by Western blot.

NY-ESO-1 vaccine group and $198.2 \pm 32.1 \mathrm{~mm}^{3}$ in the $\mathrm{NACH}$ vaccine group $(P<0.05)$. In addition, the survival fraction of tumor-bearing mice was significantly prolonged, with eight of ten mice still surviving in the NACH group on day 90. These results demonstrated that $\mathrm{NACH}$ vaccine can induce a more effective anti-tumor immune response than noncombined vaccine, which was sufficient to retard the tumor growth and prolong the survival time of tumorbearing mice.

In the therapeutic model (Figure $3 \mathrm{~A}$ and $\mathrm{B}$ ), the mean tumor volume in the mice of inoculated NS-1 mouse multiple myeloma cells was, respectively, 1401.68 $\pm 113.72 \mathrm{~mm}^{3}, \quad 592.88 \pm 76.61 \mathrm{~mm}^{3}, \quad$ and 393.6 $\pm 55.68 \mathrm{~mm}^{3}$, in the NS group, NY-ESO-1 vaccine group, and NACH group on day 21. Furthermore, seven of ten tumor-bearing mice still survived in the $\mathrm{NACH}$ vaccine group on day 90. However, all of the tumor-bearing mice died within 40 days in the NS group. The results indicated that vaccination with NY-alum-CpG-HH2 complex could inhibit tumorigenesis and increase the survival time of tumor-bearing mice effectively.
A

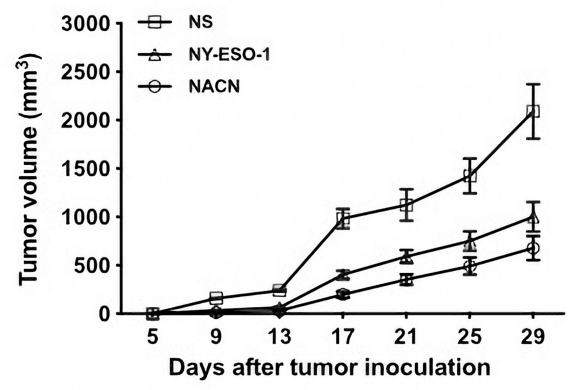

B

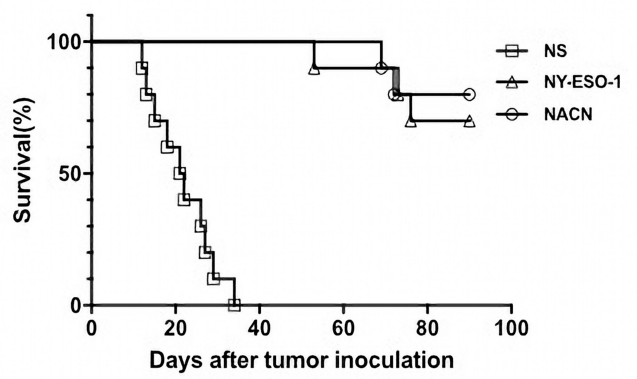

Figure 2 Tumor volumes and survival fractions of tumor-bearing mice in a prophylactic model. (A) The tumor volume of mice in a prophylactic model; (B) The survival time of tumor-bearing mice in a prophylactic model. 
A

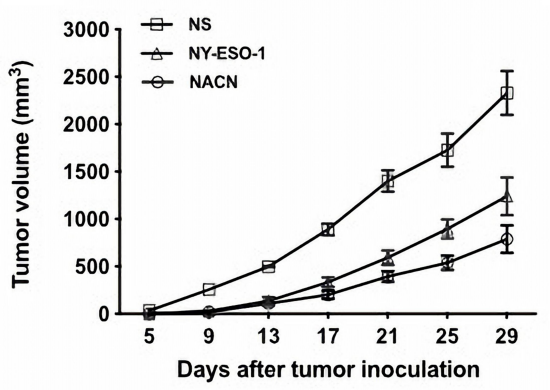

B

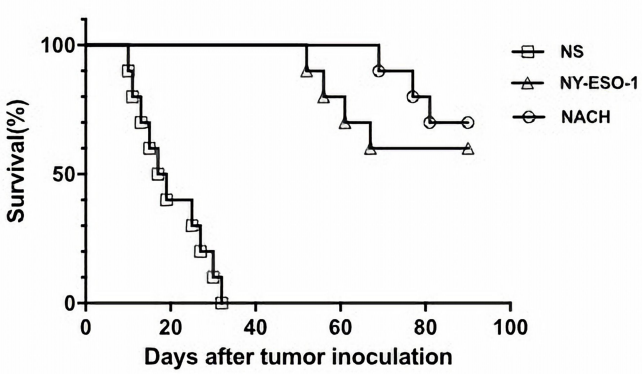

Figure 3 Tumor volumes and survival fractions of tumor-bearing mice in a therapeutic model. (A) The tumor volume of mice in a therapeutic model; (B) The survival of bearing-tumor mice in a therapeutic model.

\section{$\mathrm{NACH}$ Vaccine Led to a Highest Antigen- Specific Antibody Immune Response}

We detected titers of the antigen-specific antibody 1 week after the third immunization using ELISA and found that there was little increase of antibody titers of NY-ESO-1 in the NS group. At the same time, NY-ESO-1 vaccine enhanced the titers of antibody significantly compared with the NS group $(P<0.05)$. As expected, in terms of the NACH vaccine group, titers of antigen-specific antibody were elevated significantly contrasted to the other groups (Figure 4) $(P<0.05)$. These results suggested that NACH vaccine resulted in an elevated humoral immune response against NY-ESO-1 in murine multiple myeloma models.

\section{$\mathrm{NACH}$ Vaccine Caused Stronger Cytotoxic T Lymphocyte (CTL)} Responses to Inhibit Tumor Growth

CTL activity was performed using the standards lactate dehydrogenase (LDH) method to assess the cell-mediated

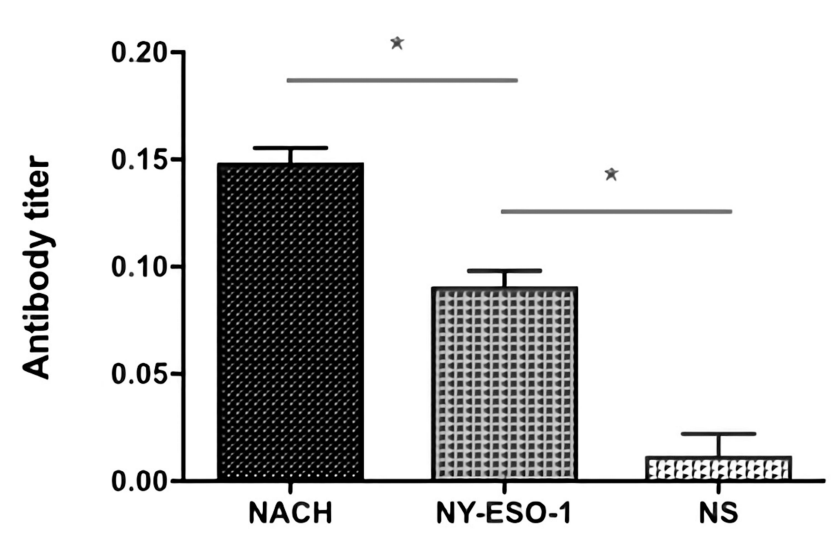

Figure 4 Detection of NY-ESO-I antibody in murine serum. The NY-ESO-I levels were detected by ELISA in the serum of mice I week after the third immunization. The levels of the NACH vaccine group were higher than that of NY-ESO-I vaccine group. $* P<0.05$. immune effect stimulated by the NACH vaccine. Splenocytes were activated and employed as effectors to identify NY-ESO- $1^{+}$NS-1 myeloma cells. CTL assays were proved by the killing rate of effector cells against target cells. The ratios of effector cells:target cells were set to $10: 1,20: 1$, and $40: 1$. Compared with $47.9 \pm 0.9 \%$ in the $\mathrm{NACH}$ vaccine group, the killing rate of the $40: 1$ ratios of effector-to-target cells were, respectively, $38.3 \pm 0.7 \%$, and $6.1 \pm 0.2 \%$ in the NY-ESO- 1 group and NS group. As expected, NACH vaccine induced remarkable tumor antigen specific cytotoxic activity $(P<0.01)$ (Figure 5$)$.

\section{$\mathrm{NACH}$ Vaccine Led to Stronger IL-4 and INF- $\gamma$ Expression in vivo}

We evaluated the INF- $\gamma$ (Th1) and IL-4 (Th2) levels in spleen lymphocytes by ELISA assay in order to determine the further cell-mediated immune responses against mice multiple myeloma inoculated NS-1. There was no obviously detectable number of INF- $\gamma / \mathrm{IL}-4$-secreting cells in the NS

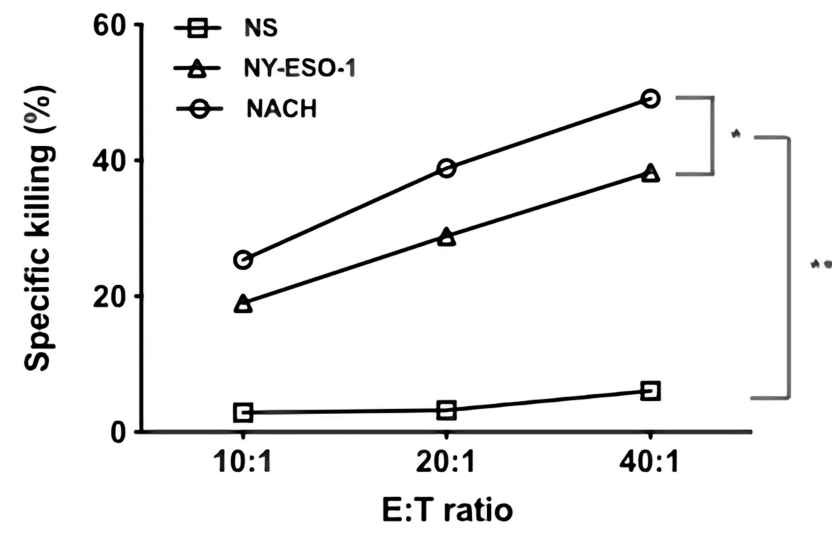

Figure 5 Killing activity of cytotoxic T lymphocyte. The cytotoxicity of splenocytes against NS-I cells was examined by LDH assay. The CTL effect of mice in the $\mathrm{NACH}$ vaccine group was highest among the groups $(P<0.05)$. $* P<0.05, * * P<0.0$ I. 


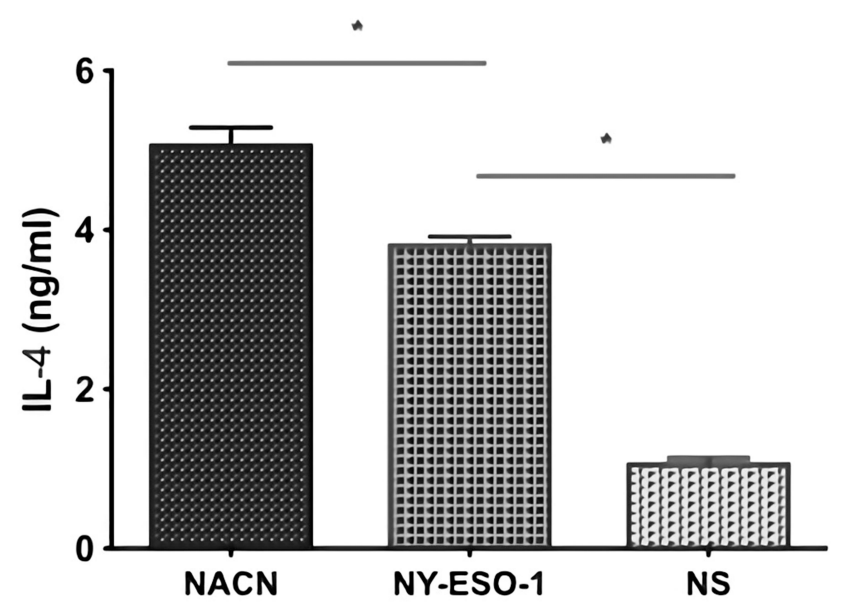

Figure 6 Detection of secreting IL-4 of the splenocytes by ELISA. $* P<0.05$.

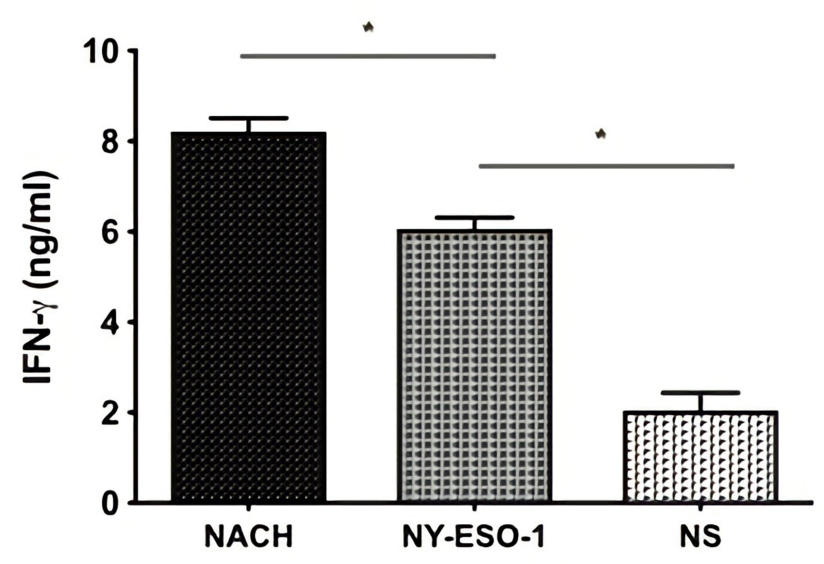

Figure 7 Detection of secreting IFN- $\gamma$ of the splenocytes by ELISA. $* P<0.05$.

group. In contrast, mice vaccination with NY-ESO-1 and $\mathrm{NACH}$ vaccine group had increased levels of INF- $\gamma / \mathrm{IL}-4$ secreting cells. Moreover, the mice vaccination with $\mathrm{NACH}$ vaccine produced the highest levels of INF- $\gamma / \mathrm{IL}-4$ levels. (Figures 6 and 7). The above results indicate that NACH vaccine can be a valid substance to induce a potent CTLs activity.

\section{$\mathrm{NACH}$ Vaccination Had No Stronger Side Effects}

Considering the safety of $\mathrm{NACH}$ vaccine, we carried out the test for its toxicity in this study. During the entire immunization process, there was no abnormal body weight (Figure 8, $\mathrm{p}>0.05$ ), appetite, hair, or activity in mice among different groups. The heart, liver, spleen, lung, and kidney from the mice of all three groups were subject to histological examinations with $\mathrm{HE}$ staining. The

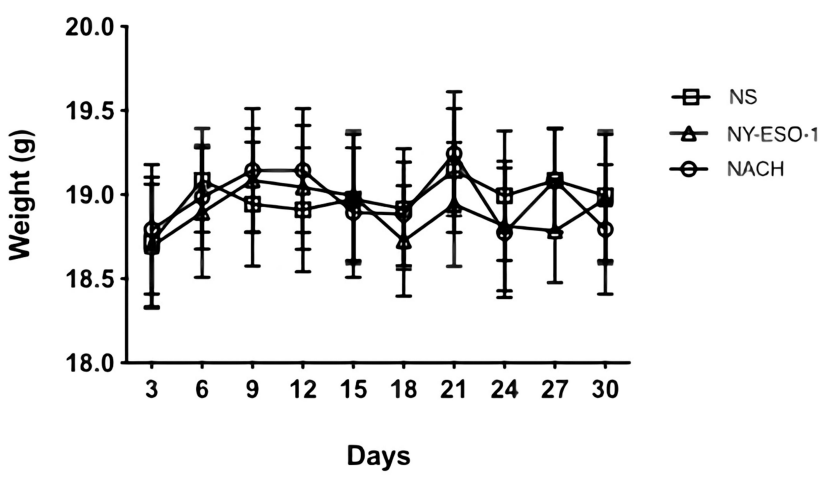

Figure 8 The body weight of mice among three groups during the whole immune procedure. There were no significant differences $(P>0.05)$.

examinations did not show any obvious pathological abnormalities (Figure 9). As a result, we confirm that $\mathrm{NACH}$ vaccine has no side-effects.

\section{Discussion}

Multiple myeloma (MM) is a cancer of malignant proliferation of plasma cells. Despite the emergence of many new drugs, MM is still an incurable disease. However, many recent researchers found cancer immunotherapy was a promising method to suppress cancer. ${ }^{33}$ The key to realize cancer immunotherapy is finding a type of antigen which is expressed in tumor tissue but not or less in normal tissue. NY-ESO-1 is prevalently expressed at the RNA and protein level not in normal tissues but in MM. ${ }^{13,19}$ As a result, NY-ESO-1 might be an excellent candidate as a tumor specific antigens (TSA) in MM. Up to now, there have been relevant literature reports about NY-ESO-1 vaccine in MM and some have already been on Phase I/II clinical trials. ${ }^{34,36}$ However, it still has not been used in the clinic because of lower immunogenicity and anti-tumor activity. ${ }^{36}$ As a result, we need further research about this tumor vaccine against NY-ESO-1 in MM. According to our experiment, NY-ESO-1 vaccine alone can inhibit tumor growth and enhance the anti-tumor immune response of the host (BALB/c mice), but the effect is not dramatic enough. So we decided to add adjuvants to the vaccine to enhance its immunogenicity and anti-tumor activity.

Alum-CpG-HH2 combinational adjuvant is a new type of immune adjuvant and it is made by mixing alum, CPG$\mathrm{ODN}$, and $\mathrm{HH} 2$ in a certain proportion. It is worth mentioning that it is a very effective immune adjuvant that significantly enhances the host's Th1-type immune response to tumor-related antigens. ${ }^{31}$ According to Tian et al's ${ }^{31}$ 


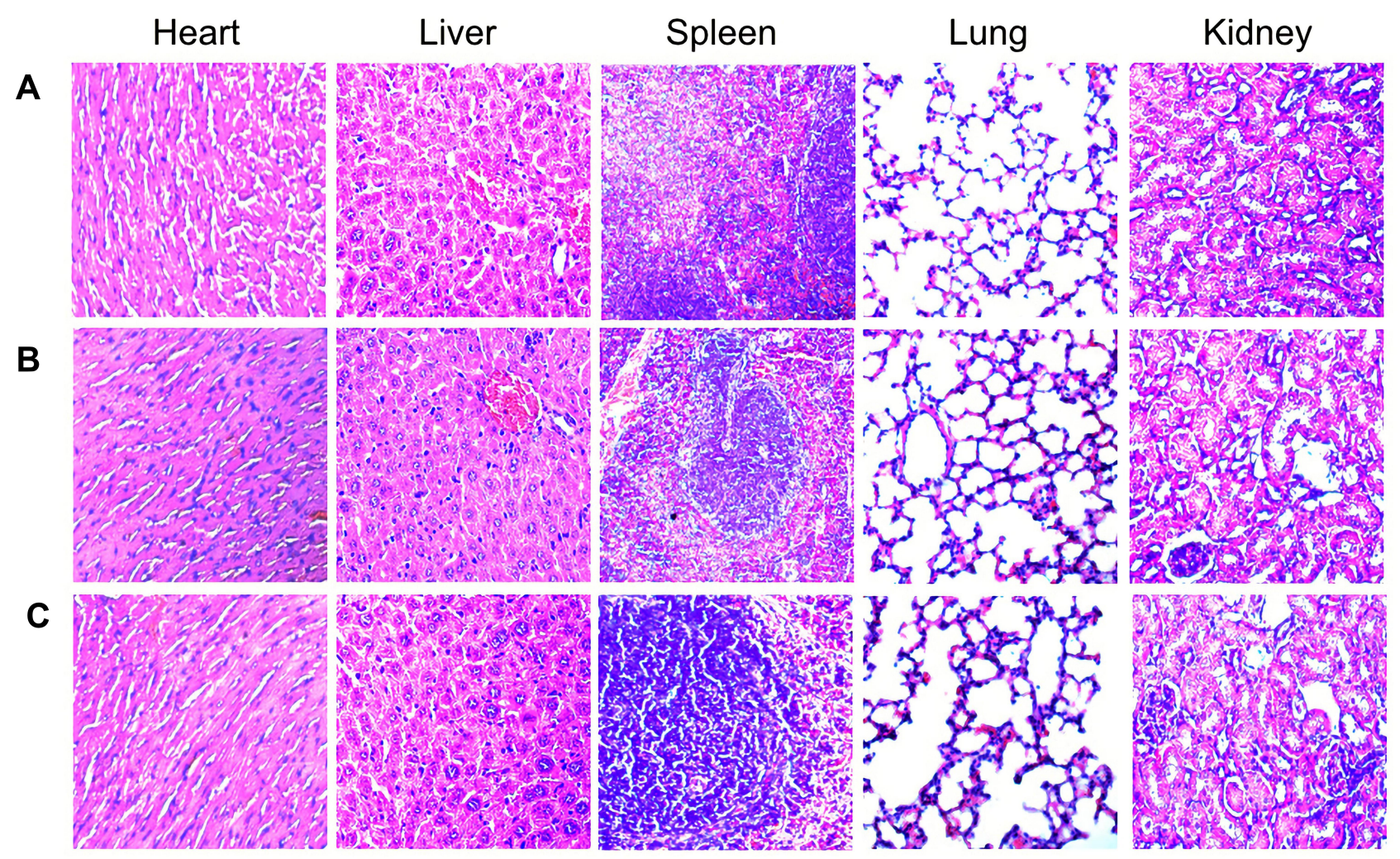

Figure 9 HE staining in tissues of heart, liver, spleen, lung, and kidney ( $\times 400)$. (A) NS group; (B) NY-ESO-I group; (C) NACH vaccine group.

research, the Alum-CpG-HH2 combinational adjuvant strengthens the body's innate immunity. In our study, we constructed alum-CpG-HH2 recombinant NY-ESO-1 protein vaccine (named $\mathrm{NACH}$ ) and found that $\mathrm{NACH}$ vaccine inhibited tumor growth and prolonged survival in mice in both the prophylactic and therapeutic groups more strongly than the NY-ESO-1 vaccine. Most notably, mice had the longest survival and the smallest tumor volume measured in the $\mathrm{NACH}$ vaccine group.

We detected titers of the antigen-specific antibody by ELISA to assess the enhancement of host humoral immunity in three groups. In the NS group, the increase of antibody titers against NY-ESO-1 was not significant. NY-ESO-1 vaccine enhanced the titers of NY-ESO-1 antibody, slightly but not significantly. As we expected, $\mathrm{NACH}$ immunized mice developed the highest levels of NY-ESO-1 antibody among the three groups. These results suggest that $\mathrm{NACH}$ vaccine can significantly induce humoral immunity against multiple myeloma. Tian et al's ${ }^{31}$ findings also suggest that vaccine combined with Alum-CpG-HH2 combinational adjuvant significantly increases the concentration of $\mathrm{IgG}$ in the blood of mice.
In order to evaluate the effect of the $\mathrm{NACH}$ vaccine on the cellular immune response, we conducted the following assays. First, we performed CTL activity and used the standards lactate dehydrogenase (LDH) method to detect the killing rate of effector cells against target cells. Compared with other groups, the killing rate of the NACH vaccine group was significantly higher. Secondly, we also evaluated the INF- $\gamma$ (Th1) and IL-4 (Th2) levels in spleen lymphocytes by ELISA assay in order to determine the further cell-mediated immune responses against mice multiple myeloma inoculated NS-1. The levels of INF- $\gamma / \mathrm{IL}-4$ secreting cells in the $\mathrm{NACH}$ group were highest among three groups. The results of the above two experiments suggest that the $\mathrm{NACH}$ vaccine activated a sufficiently strong cellular immunity against MM. Previous reports have also confirmed that there are multiple epitopes of NY-ESO-1 peptide chain recognized by $\mathrm{T}$ lymphocytes and these epitopes can effectively induce adaptive CTL response in NY-ESO-1 positive patients. In addition, purified recombinant NY-ESO-1 protein vaccines can significantly trigger the $\mathrm{CD}^{+} \mathrm{T}$ cell response and activate dendritic cells regardless of HLA restriction. As 
a whole, this purified recombinant NY-ESO-1 protein vaccine is effective in humoral and cellular immunity. ${ }^{34}$

In addition, the safety of vaccines has always been a concern. Therefore, during the course of the experiment, we performed a rigorous physical examination of the mice, and then we did not find any abnormal situation of appetite, hair, and activity in mice among different groups. In addition, we also performed an autopsy on the mice after the experiment, and we did not observe pathological abnormalities of the heart, liver, spleen, lung, and kidney from the mice of all three groups. It turned out that our vaccine had no obvious side-effects on mice.

To sum up, our data demonstrated that the NACH vaccine is a safe, effective, and promising vaccine, which can effectively trigger efficient humoral and cellular tumor-specific immune responses and inhibit tumorigenesis and progression in murine multiple myeloma. The results in this study may provide a new strategy for immunotherapy of multiple myeloma.

\section{Acknowledgment}

This project was supported by the foundation of health commission of Sichuan province.

\section{Disclosure}

The authors report no conflicts of interest for this work.

\section{References}

1. Krstevska SB, Sotirova T, Balkanov T, Genadieva-Stavric S. Treatment approach of nontransplant patients with multiple myeloma. Mater Sociomed. 2014;26:348-351. doi:10.5455/msm.20 14.26.348-351

2. Liu TT, Wu Y, Niu T. Human DKK1 and human HSP70 fusion DNA vaccine induces an effective anti-tumor efficacy in murine multiple myeloma. Oncotarget. 2018;9(1):178-191. doi:10.18632/oncotarget. 23352

3. Zhang L, Gotz M, Hofmann S, Greiner J. Immunogenic targets for specific immunotherapy in multiple myeloma. Clin Dev Immunol. 2012;2012:820394. doi:10.1155/2012/820394

4. Anderson KC, Carrasco RD. Pathogenesis of myeloma. Annu Rev Pathol. 2011;6:249-274. doi:10.1146/annurev-pathol-011110-130249

5. Yi Q. Novel immunotherapies. Cancer J. 2009;15:502-510. doi:10.1097/PPO.0b013e3181c51f0d

6. Sharma P, Wagner K, Wolchok JD, Allison JP. Novel cancer immunotherapy agents with survival benefit: recent successes and next steps. Nat Rev Cancer. 2011;11:805-812. doi:10.1038/nrc3153

7. Mellman I, Coukos G, Dranoff G. Cancer immunotherapy comes of age. Nature. 2011;480:480-489. doi:10.1038/nature10673

8. Barlogie B, Shaughnessy J, Tricot G, et al. Treatment of multiple myeloma. Blood. 2004;103:20-32. doi:10.1182/blood-2003-04-1045

9. Lokhorst HM, Schattenberg A, Cornelissen JJ, et al. Donor lymphocyte infusions for relapsed multiple myeloma after allogeneic stem-cell transplantation: predictive factors for response and long-term outcome. J Clin Oncol. 2000;18:3031-3037. doi:10.1200/JCO.2000.18.16.3031
10. Barlogie B, Tricot G, Anaissie E, et al. Thalidomide and hematopoietic-cell transplantation for multiple myeloma. $N$ Engl $J$ Med. 2006;354:1021-1030. doi:10.1056/NEJMoa053583

11. Chen YT, Scanlan MJ, Sahin U, et al. A testicular antigen aberrantly expressed in human cancers detected by autologous antibody screening. Proc Natl Acad Sci (USA). 1997;94:1914-1918. doi:10.1073/pnas.94.5.1914

12. Lethe B, Lucas S, Michaux L, et al. LAGE-1, a new gene with tumor specificity. Int J Cancer. 1998;76:903-908. doi:10.1002/(SICI)10970215(19980610)76:6<903::AID-IJC22>3.0.CO;2-1

13. Jungbluth AA, Chen YT, Stockert E, et al. Immunohistochemical analysis of NY-ESO- 1 antigen expression in normal and malignant human tissues. Int $J$ Cancer. 2001;92:856-860. doi:10.1002/ijc.1282

14. Sugita Y, Wada H, Fujita S, et al. NY-ESO-1 expression and immunogenicity in malignant and benign breast tumors. Cancer Res. 2004;64:2199-2204. doi:10.1158/0008-5472.CAN-03-3070

15. Nakada T, Noguchi Y, Satoh S, et al. NY-ESO-1 mRNA expression and immunogenicity in advanced prostate cancer. Cancer Imm. 2003;3:10.

16. Odunsi K, Jungbluth AA, Stockert E, et al. NY-ESO-1 and LAGE-1 cancer-testis antigens are potential targets for immunotherapy in epithelial ovarian cancer. Cancer Res. 2003;63:6076-6083.

17. Maio M, Coral S, Sigalotti L, et al. Analysis of cancer/testis antigens in sporadic medullary thyroid carcinoma: expression and humoral response to NY-ESO-1. J Clin Endocrinol Metab. 2003;88: 748-754. doi:10.1210/jc.2002-020830

18. Sharma P, Gnjatic S, Jungbluth AA, et al. Frequency of NY-ESO-1 and LAGE-1 expression in bladder cancer and evidence of a new NYESO-1 T-cell epitope in a patient with bladder cancer. Cancer Immun. 2003;3:19. doi:10.1186/2051-1426-3-S2-O19

19. Wang Y, Wu XJ, Zhao AL, et al. Cancer/testis antigen expression and autologous humoral immunity to NY-ESO-1 in gastric cancer. Cancer Immun. 2004;4:11.

20. MacLeod MK, McKee AS, David A, et al. Vaccine adjuvants aluminum and monophosphoryl lipid A provide distinct signals to generate protective cytotoxic memory CD8 T cells. Proc Natl Acad Sci U S A. 2011;108:7914-7919. doi:10.1073/pnas.1104588108

21. Gupta RK. Aluminum compounds as vaccine adjuvants. $A d v$ Drug Deliv Rev. 1998;32:155-172. doi:10.1016/S0169-409X(98) 00008-8

22. Mazloomi E, Jazani NH, Shahabi S. A novel adjuvant, mixture of alum and the beta-adrenergic receptor antagonist propranolol, elicits both humoral and cellular immune responses for heat-killed salmonella typhimurium vaccine. Vaccine. 2012;30:2640-2646. doi:10.10 16/j.vaccine.2012.02.017

23. Disis ML, Bernhard H, Shiota FM, et al. Granulocyte-macrophage colony-stimulating factor: an effective adjuvant for protein and peptide-based vaccines. Blood. 1996;88:202-210. doi:10.1182/ blood.V88.1.202.202

24. Meng L, Shi H, Zhang H, et al. Effective inhibition of melanoma tumorigenesis and growth via a new complex vaccine based on NY-ESO-1-alum-polysaccharide-HH2. Mol Cancer. 2014;13:1-5. doi:10.1186/1476-4598-13-1

25. Veenstra JJ, Gibson HM, Littrup PJ, et al. Cryotherapy with concurrent $\mathrm{CpG}$ oligonucleotide treatment controls local tumor recurrence and modulates HER2/neu immunity. Cancer Res. 2014;74:54 09-5420. doi:10.1158/0008-5472.CAN-14-0501

26. Chang LS, Leng CH, Yeh YC, et al. Toll-like receptor 9 agonist enhances anti-tumor immunity and inhibits tumor-associated immunosuppressive cells numbers in a mouse cervical cancer model following recombinant lipoprotein therapy. Mol Cancer. 2014;13:60. doi:10.1186/1476-4598-13-60

27. Xia Y, Gupta GK, Castano AP, Mroz P, Avci P, Hamblin MR. CpG oligodeoxynucleotide as immune adjuvant enhances photodynamic therapy response in murine metastatic breast cancer. J Biophotonics. 2014;7:897-905. doi:10.1002/jbio.201300072 
28. Easton DM, Nijnik A, Mayer ML, Hancock RE. Potential of immunomodulatory host defense peptides as novel anti-infectives. Trends Biotechnol. 2009;27:582-590. doi:10.1016/j.tibtech.2009.07.004

29. Bowdish DM, Davidson DJ, Lau YE, Lee K, Scott MG, Hancock RE. Impact of LL-37 on anti-infective immunity. $J$ Leukoc Biol. 2005;77:451-459. doi:10.1189/jlb.0704380

30. Kindrachuk J, Jenssen $H$, Elliott $M$, et al. A novel vaccine adjuvan comprised of a synthetic innate defence regulator peptide and $\mathrm{CpG}$ oligonucleotide links innate and adaptive immunity. Vaccine. 2009;27:4662-4671. doi:10.1016/j.vaccine.2009.05.094

31. Tian Y, Li M, Yu C, et al. The novel complex combination of alum, CpG ODN and HH2 as adjuvant in cancer vaccine effectively suppresses tumor growth in vivo. Oncotarget. 2017;8(28):45951-45964. doi:10.18632/oncotarget.17504

32. Niyonsaba F, Madera L, Afacan N, Okumura K, Ogawa H, Hancock RE. The innate defense regulator peptides IDR-HH2, IDR-1002, and IDR-1018 modulate human neutrophil functions. J Leukoc Biol. 2013;94:159-170. doi:10.1189/jlb.1012497
33. Torre LA, Bray F, Siegel RL, Ferlay J, Lortet-Tieulent J, Jemal A. Global cancer statistics, 2012. CA Cancer J Clin. 2015;65:87-108. doi:10.3322/caac. 21262

34. Szmania S, Tricot G, Van Rhee F. NY-ESO-1 immunotherapy for multiple myeloma. Leuk Lymphoma. 2006;47(10):2037-2048. doi:10.1080/10428190600742292

35. van Rhee F, Szmania SM, Zhan F, et al. NY-ESO-1 is highly expressed in poor-prognosis multiple myeloma and induces spontaneous humoral and cellular immune responses. Blood. 2005;105:3939-3944. doi:10.1182/blood-2004-09-3707

36. Rapoport AP, Stadtmauer EA, Binder-Scholl GK, et al. NY-ESO -1-specific TCR-engineered $\mathrm{T}$ cells mediate sustained antigen-specific anti-tumor effects in myeloma. Nat Med. 2015;21 (8):914-921. doi:10.1038/nm.3910

\section{Publish your work in this journal}

OncoTargets and Therapy is an international, peer-reviewed, open access journal focusing on the pathological basis of all cancers, potential targets for therapy and treatment protocols employed to improve the management of cancer patients. The journal also focuses on the impact of management programs and new therapeutic agents and protocols on patient perspectives such as quality of life, adherence and satisfaction. The manuscript management system is completely online and includes a very quick and fair peer-review system, which is all easy to use. Visit http://www.dovepress.com/ testimonials.php to read real quotes from published authors. 